\title{
NUMERICAL SIMULATIONS OF BUOYANCY EFFECTS IN LOW DENSITY GAS JETS
}

\author{
R. P. Satti ${ }^{*}$ K. S. Pasumarthi" and A. K. Agrawal $^{\#}$ \\ School of Aerospace and Mechanical Engineering \\ University of Oklahoma, Norman, OK-73019, USA
}

\begin{abstract}
This paper deals with the computational analysis of buoyancy effects in the near field of an isothermal helium jet injected into quiescent ambient air environment. The transport equations of helium mass fraction coupled with the conservation equations of mixture mass and momentum were solved using a staggered grid finite volume method. Laminar, axisymmetric, unsteady flow conditions were considered for the analysis. An orthogonal system with non-uniform grids was used to capture the instability phenomena. Computations were performed for Earth gravity and during transition from Earth to different gravitational levels. The flow physics was described by simultaneous visualizations of velocity and concentration fields at Earth and microgravity conditions. Computed results were validated by comparing with experimental data substantiating that buoyancy induced global flow oscillations present in Earth gravity are absent in microgravity. The dependence of oscillation frequency and amplitude on gravitational forcing was presented to further quantify the buoyancy effects.
\end{abstract}

Keywords: Buoyancy, Flow computations, Flow Instability.

\section{INTRODUCTION}

Low density gas jet flows are encountered in many engineering and technical applications such as smokestack discharge, fuel leak, space propulsion, aerosol spray, jets entering mixing chamber, ventilation jets and many other environmental issues relating to atmosphere and hydrosphere. Jets are termed as buoyant for Richardson number, $\operatorname{Ri}=\operatorname{gd}\left(\rho_{\infty}-\rho\right) / \rho \mathrm{V}^{2}>1.0$ and inertial for $\mathrm{Ri}<<1.0$. The present study examines the near field flow structure of a buoyant helium jet injected into quiescent ambient air environment.

\footnotetext{
* Graduate Research Assistant
}

\# Member AIAA, Associate Professor

Copyright@2004 by A.K. Agrawal. Published by the American Institute of Aeronautics and Astronautics, Inc., with permission.
Several studies ${ }^{1-6}$ have focused on the far field behavior of jets. However, the near field flow stability has direct influence on the entrainment and flow pattern in the far field. Self-excited oscillations in the near injector region of low-density gas jets have been studied extensively. Subbarao and Cantwell ${ }^{7}$ observed highly periodic oscillations in the near field of helium jets injected into a co-flow air environment for Ri between 0.5 and 6.0. The Strouhal number, $\mathrm{St}=\mathrm{fd} / \mathrm{V}$ correlated with Richardson number for $\mathrm{Ri}>1.0$ indicating buoyancy dependent instability mode. Similar experiments were conducted by Cetegen ${ }^{8-10}$ for axisymmetric and planar plumes, whereby buoyancy induced toroidal vortical structures contaminating the primary jet flow were observed. Agrawal and coworkers $^{11-12}$ conducted experiments to characterize the flow instability in terms of concentration measurements across the whole field of a helium jet injected into quiescent air. Experiments by Yep et al. ${ }^{11}$ demonstrated that flow oscillations were absent in the microgravity environment of the $2.2 \mathrm{~s}$ drop tower.

Few computational studies have also been a part of low-density jet instability literature. Mell et al. ${ }^{13}$ performed numerical simulations of helium jet in air and found that the computed oscillation frequency matched with experimentally obtained value by Hamins et $\mathrm{a}^{14}$. Recently, Soteriou ${ }^{15}$ revealed that the instability characteristics in buoyant planar plumes compared well with experiments, providing insight into the role played by viscous and buoyancy forces in establishing oscillating or non-oscillating modes.

The above studies provided extensive details relating to flow instability, attributed to buoyancy dominating the flow field. Although the instability in high Richardson number helium jets is attributed to buoyancy, direct physical evidence with respect to concurrent visualizations of velocity and concentration fields is lacking in the present literature. 
If the instability is indeed caused by buoyancy, the near field flow structure of the jet will change significantly when buoyancy is removed. Thus, quantitative data on the spatial and temporal evolutions of the instability, length and time scales of the oscillating mode and its effects on the mean flow are needed in Earth gravity and microgravity conditions to delineate gravitational effects in buoyant jets.

Although microgravity experiments ${ }^{11}$ have quantified buoyancy effects and provided details of the concentration field, results are devoid of the flow structure details. Simultaneous visualization of velocity and concentration fields is desired to fully understand the oscillating instability and consequent flow structure of the jet. Owing to the experimental limitations in obtaining such measurements, especially in microgravity, the present study utilizes computational analysis to simulate helium jet in air. Features of the jet are described in both Earth gravity and microgravity. In the following section, we provide details relating to the governing equations, boundary and initial conditions, and the computational procedure. Results obtained at Earth gravity and microgravity conditions are discussed subsequently.

\section{NUMERICAL FORMULATION}

\section{Governing Equations}

The governing equations for laminar, unsteady and axisymmetric flow are expressed in the cylindrical coordinate system as follows:

$$
\begin{aligned}
& \frac{\partial \rho}{\partial t}+\frac{\partial}{\partial r}(\rho v r)+\frac{\partial}{\partial z}(\rho r w)=0 \\
& \frac{\partial(\rho \phi)}{\partial t}+\frac{\partial}{\partial r}(\rho v r \phi)+\frac{\partial}{\partial z}(\rho r w \phi)=\frac{\partial}{\partial r}\left(\Gamma^{\phi} r \frac{\partial \phi}{\partial r}\right)+\frac{\partial}{\partial z}\left(\Gamma^{\phi} r \frac{\partial \phi}{\partial z}\right)+S^{\phi}
\end{aligned}
$$

where $\rho$ represents density; and $\mathrm{w}$ and $\mathrm{v}$ are, respectively, the axial and radial components of the velocity. Equation 1 represents the continuity equation and Equation 2 is the general transport equation, which reduces to momentum, or species conservation equation depending on the variable $\phi$. The terms $\Gamma^{\phi}$ and $\mathrm{S}^{\phi}$ in the transport equation represent the diffusion coefficient and source term of the variable $\phi$. The coefficients and source terms appearing in Equation 2 are listed in Table 1. In this study, the temperature and pressure variations are considered to be negligible and the jet flow is assumed to be a binary fluid system.
Table 1. Coefficients and source terms in Equation 2.

\begin{tabular}{|c|c|c|}
\hline$\phi$ & $\Gamma^{\phi}$ & $\mathrm{S}^{\phi}$ \\
\hline $\mathrm{w}$ & $\mu$ & $-\mathrm{r} \frac{\partial \mathrm{p}}{\partial \mathrm{z}}+\mathrm{r}\left(\rho_{\infty}-\rho\right) \mathrm{g}+\frac{\partial}{\partial \mathrm{z}}\left(\mathrm{r} \mu \frac{\partial \mathrm{w}}{\partial \mathrm{z}}\right)+\frac{\partial}{\partial \mathrm{r}}\left(\mathrm{r} \mu \frac{\partial \mathrm{v}}{\partial \mathrm{z}}\right)-\frac{2}{3}\left[\frac{\partial}{\partial \mathrm{z}}\left(\mathrm{r} \mu \frac{\partial \mathrm{v}}{\partial \mathrm{r}}\right)+\frac{\partial}{\partial \mathrm{z}}\left(\mathrm{r} \mu \frac{\partial \mathrm{w}}{\partial \mathrm{z}}\right)\right]$ \\
\hline $\mathrm{v}$ & $\mu$ & $-\mathrm{r} \frac{\partial \mathrm{p}}{\partial \mathrm{r}}-\frac{2}{3} \mu \frac{\mathrm{v}}{\mathrm{r}}+\frac{\partial}{\partial \mathrm{z}}\left(\mathrm{r} \mu \frac{\partial \mathrm{w}}{\partial \mathrm{r}}\right)+\frac{\partial}{\partial \mathrm{r}}\left(\mathrm{r} \mu \frac{\partial \mathrm{v}}{\partial \mathrm{r}}\right)-\frac{2}{3}\left[\frac{\partial}{\partial \mathrm{r}}\left(\mathrm{r} \mu \frac{\partial \mathrm{v}}{\partial \mathrm{r}}\right)+\frac{\partial}{\partial \mathrm{r}}\left(\mathrm{r} \mu \frac{\partial \mathrm{w}}{\partial \mathrm{z}}\right)\right]$ \\
\hline $\mathrm{f}$ & $\mathrm{D}_{\mathrm{b}}$ & 0 \\
\hline
\end{tabular}

\section{Thermo-Physical Properties}

The density was computed from the incompressible ideal gas law as follows:

$$
\rho=\frac{P_{o p}}{R T \sum_{i} \frac{Y_{i}}{M_{w, i}}}=\frac{P_{o p}}{R T\left[\frac{Y_{h e}}{M_{w, h e}}+\frac{Y_{a i r}}{M_{w, a i r}}\right]}
$$

The dynamic viscosity was calculated using the Wilke's mixture averaged formula ${ }^{16}$ as follows:

$$
\begin{aligned}
\mu=\sum_{i=1}^{n} \frac{Y_{i} \mu_{i}}{\sum_{j=1}^{n} Y_{j} \phi_{i j}}= & \frac{Y_{h e} \mu_{h e}}{Y_{h e} \phi_{11}+Y_{a i r} \phi_{12}}+\frac{Y_{a i r} \mu_{a i r}}{Y_{h e} \phi_{21}+Y_{a i r} \phi_{22}} \\
\text { with } \quad \phi_{i j} & =\frac{\left[1+\left(\frac{\mu_{i}}{\mu_{j}}\right)^{1 / 2}\left(\frac{M_{w, j}}{M_{w, i}}\right)^{1 / 4}\right]^{2}}{\left[8\left(1+\frac{M_{w, i}}{M_{w, j}}\right)\right]^{1 / 2}}
\end{aligned}
$$

where subscripts 1 and 2 represent helium and air, respectively. The binary diffusion coefficient of helium into air, $\mathrm{D}_{\mathrm{b}}$ was specified as a constant.

\section{Operating and Boundary Conditions}

Figure 1 shows the computational domain extending $15 \mathrm{~d}$ in the axial direction and $6 \mathrm{~d}$ in the radial direction where $\mathrm{d}=31.8 \mathrm{~mm}$ is the tube inside diameter. The upstream boundary was placed at a distance of $1 \mathrm{~d}$ from the jet exit plane. Symmetric boundary condition was imposed along the jet centerline. At the tube inlet, the velocity profile was fully developed and the helium mole fraction was unity. The jet Reynolds number was specified as 300 corresponding to Richardson number of 1.52. No slip and zero mass diffusion conditions were imposed at the tube walls. At the exit boundary, pressure outlet condition was implemented instead of 
outflow conditions to account for the backflow. Figure 1 shows pressure conditions imposed at other boundaries. The operating pressure and temperature in the domain were specified, respectively, as $1 \mathrm{~atm}$ and $300 \mathrm{~K}$.

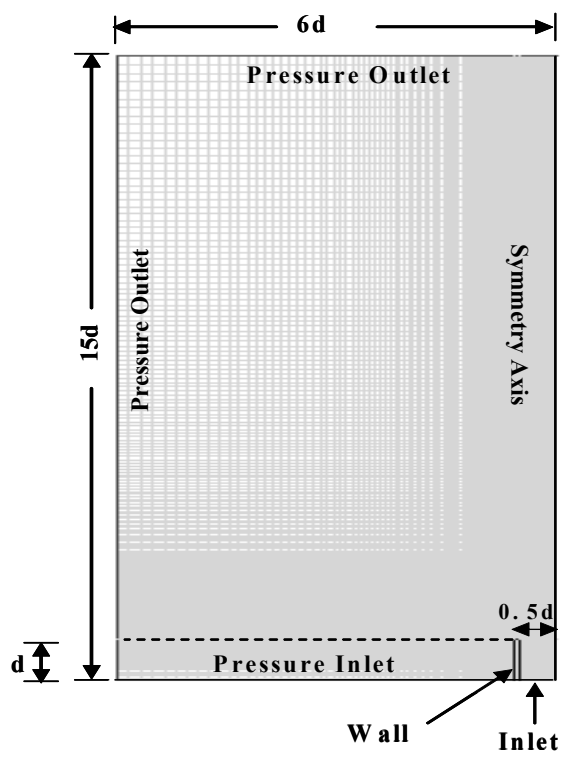

Figure 1. Computational domain.

\section{$\underline{\text { Computational Details }}$}

The grid was generated by splitting the computational domain into five sub regions. An orthogonal nonuniform grid system was used. The grid elements in the radial direction were concentrated in the oscillatory zone. The grid size was increased gradually in the axial direction. The governing equations were solved sequentially using a segregated approach ${ }^{17}$. Since the governing equations are coupled, several iterations of the solution loop were performed before a converged solution was obtained. The pressure velocity coupling was achieved using the SIMPLEC algorithm.

\section{RESULTS AND DISCUSSION}

Numerical simulations were performed for the isothermal helium jet injected into the ambient air environment with the objective of isolating gravitational effects in the near field. First, grid sensitivity analysis was performed using different sizes of grids and the solution was found to be independent of the grid structure. Based on the analysis, a $260 \times 95$ grid was chosen for computations. In the following sections, we provide details pertaining to the self excited instability observed in the helium jet at Earth gravity conditions. Subsequently, the near field flow physics is explained as the jet undergoes change to microgravity conditions. Further insight is gained from the parametric study by varying the gravitational level. The change in the buoyant structure of the jet is illustrated from vortex convection velocity, and flow oscillation frequency and amplitude plots.

\section{Self-Excited Oscillatory Structure in Earth Gravity}

Periodic oscillations occur in the near field of the helium jet at Earth gravity conditions owing to buoyancy induced acceleration of the jet fluid. To illustrate this phenomenon, Figure 2 shows a sequence (1/73s apart) of helium mole percentage contours superimposed with velocity vectors. The jet boundary is demarcated by the $60-70 \%$ concentration level and the vortex core is represented by a black dot. At $\mathrm{t}=0.0 \mathrm{~s}$, buoyancy accelerates the jet core, which contracts to conserve the mass. The net result is entrainment of ambient fluid illustrated by the flow recirculation representing the vortex. In the next plot at $t=1 / 73 \mathrm{~s}$, the vortex is characterized by larger recirculation region accompanied with greater contraction of the jet boundary, evident from the indentation in the concentration field. Subsequently, between $t=2 / 73 \mathrm{~s}$ and $4 / 73 \mathrm{~s}$, the vortex convects downstream while growing in size to further contaminate the jet core.

The vortex formation is accompanied by momentum transfer from the jet core to the vortex. This feature is evident in Figure 2 wherein vectors downstream of the vortex have higher magnitude than those upstream before interacting with the jet core. As the straining effect of the vortex diminishes in the upstream region, the jet expands at the exit inducting buoyant jet fluid to initiate another vortex at $\mathrm{t}=5 / 73 \mathrm{~s}$ to repeat the oscillation cycle. The above results illustrate self excited periodicity in the jet at a frequency of $14.6 \mathrm{~Hz}$, indicating both qualitative and quantitative agreement with experiments ${ }^{11}$. In the following sub-section, our study is focused on delineating buoyancy effects by simulating the jet flow in the absence of gravity.

\section{$\underline{\text { Transition from Earth to Microgravity Conditions }}$}

Computations were performed to visualize the jet flow during change from Earth to microgravity conditions and to depict how the jet flow structure adjusts itself in the absence of buoyancy driven forces. As a first step, the simulation results were compared with experiments $^{11}$ to validate the computational model. Figure 3 shows a series of computational-experimental helium mole fraction contour plots during transition from Earth gravity to microgravity. Note that the experimental data were taken from Yep et $\mathrm{al}^{11}$. 


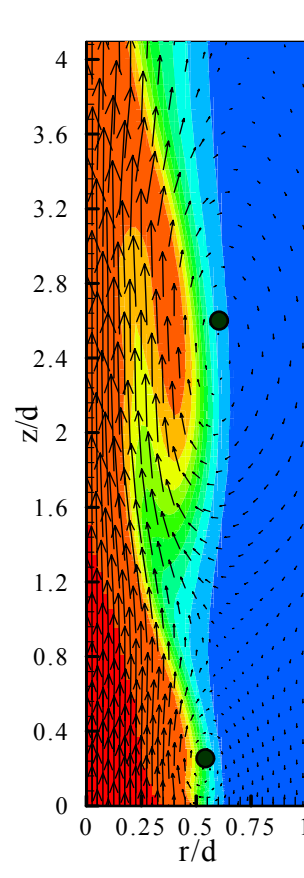

(a)

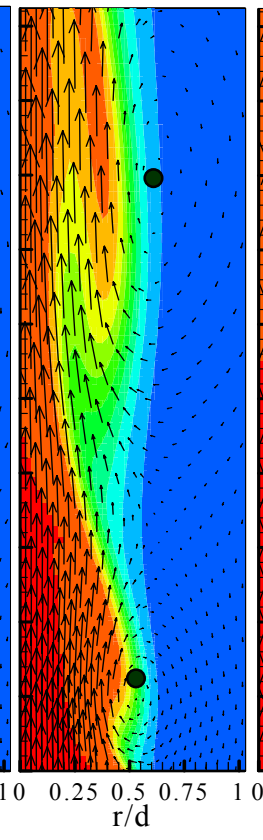

(b)

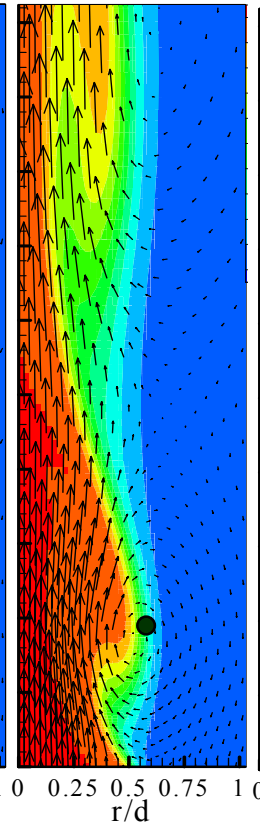

(c)

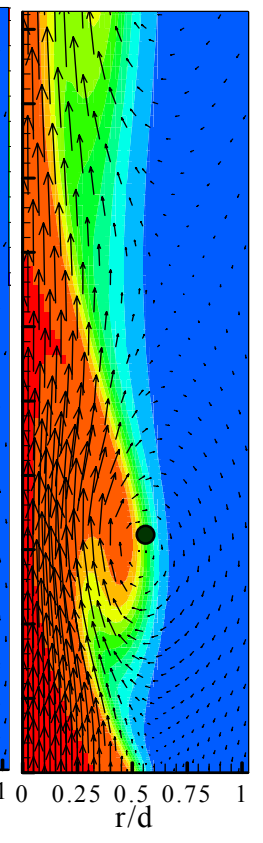

(d)

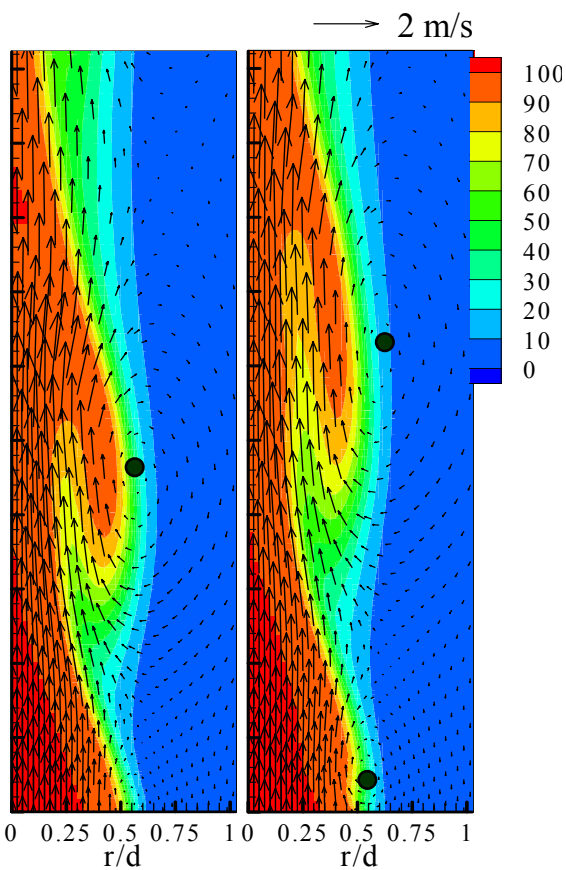

(e) (f)

Figure 2. Contours of helium mole percentage superimposed by velocity vectors during an oscillation cycle (left to right) in Earth gravity (a) $t=0.0 \mathrm{~s}$; (b) $t=1 / 73 \mathrm{~s}$; (c) $\mathrm{t}=2 / 73 \mathrm{~s}$; (d) $t=3 / 73 \mathrm{~s}$; (e) $t=4 / 73 \mathrm{~s}$; (f) $t=5 / 73 \mathrm{~s}$.

At $\mathrm{t}=0.0 \mathrm{~s}$, we observe a vortex located at $\mathrm{z} / \mathrm{d}=0.7$, indicated by the indentation in the concentration level of $50 \%$. With the initiation of microgravity, between $\mathrm{t}=1 / 60 \mathrm{~s}$ and $2 / 60 \mathrm{~s}$, the vortex convects downstream as the jet expands in the near exit region, similar to experiments ${ }^{11}$. At $\mathrm{t}=3 / 60 \mathrm{~s}$, the jet has expanded at the exit to $\mathrm{r} / \mathrm{d}=0.7$. The vortex located at $\mathrm{z} / \mathrm{d}=1.5$ has weakened as seen from the diminishing nature of the indentation and decrease in the curvature of concentration profile. Subsequently, the jet widens gradually throughout the near field with weakening and diminishing of oscillations as steady microgravity conditions are reached. The helium mole fraction contours in microgravity are straight lines reminiscent of a non-buoyant jet. The above mentioned features replicate similar phenomena observed in experiments ${ }^{11}$ wherein the self-excited oscillations gradually subsided after microgravity condition was initiated. Judging from the plots in Figure 3, it was inferred that the computations accurately reproduced the experiments proving the model's reliability in predicting the flow physics during change from Earth gravity to microgravity.

Figure 4 shows the contours of helium mole percentage overlapped by velocity vectors during transition from Earth to microgravity to highlight the interaction of the flow field with the concentration field. At $\mathrm{t}=0.0 \mathrm{~s}$, a vortex with the core located at $\mathrm{z} / \mathrm{d}=0.7$, characterizes the entrainment of ambient fluid into the helium jet. At this instant, the jet width near the exit extends to $\mathrm{r} / \mathrm{d}=0.6$. After the initiation of microgravity, at $\mathrm{t}=2 / 60 \mathrm{~s}$, the jet has expanded at the exit to $r / d=0.7$. The vortex convects downstream with its core located at about $\mathrm{z} / \mathrm{d}=1.8$. Upstream of the vortex, we observe an irregular distribution of the flow vectors together with distortions around the vortex core region. This behavior is attributed to the flow adjusting to the sudden decay in buoyancy induced acceleration along the jet core region. In the next plot at $t=4 / 60 \mathrm{~s}$, the weakened vortex moves away from the field of view as another vortex is initiated at the jet exit. Observe that the vortex core is located close to the $90 \%$ concentration level with the jet expanding to $\mathrm{r} / \mathrm{d}=0.75$. Though buoyancy forces are absent, we observe vortical motion near the exit possibly because of the residual effects.

At $\mathrm{t}=8 / 60 \mathrm{~s}$, we observe that the recirculation and entrainment have decreased with the jet continually expanding near the exit. Similar features are exhibited at $\mathrm{t}=10 / 60 \mathrm{~s}$ with flow approaching steady conditions, whereby entrainment is dominated by diffusion. Finally, at $\mathrm{t}=2.0 \mathrm{~s}$, the jet has assumed a steady columnar shape. Higher velocities persist in the jet core and the velocity decreases radially as the jet mixes with the ambient air by molecular diffusion. The vorticity magnitude (not shown) decreases when buoyancy was removed, signifying reduction in mixing of helium and air by entrainment. Further information about the flow structure is provided by the spatio-temporal plots. 


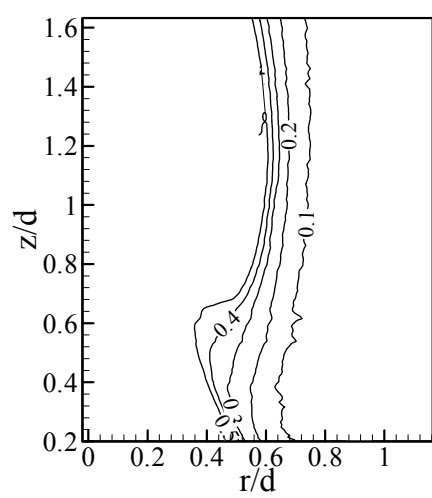

1(a)

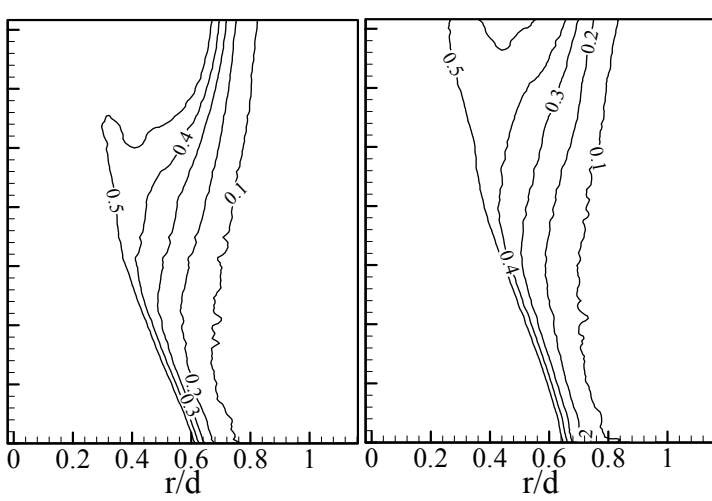

1(b)

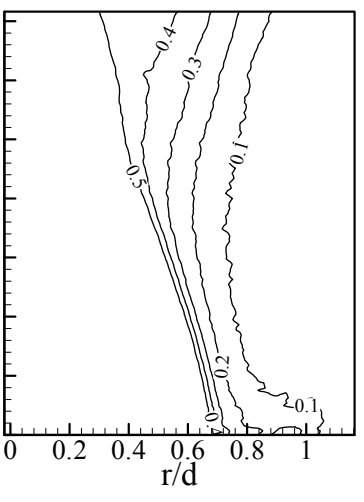

1(d)

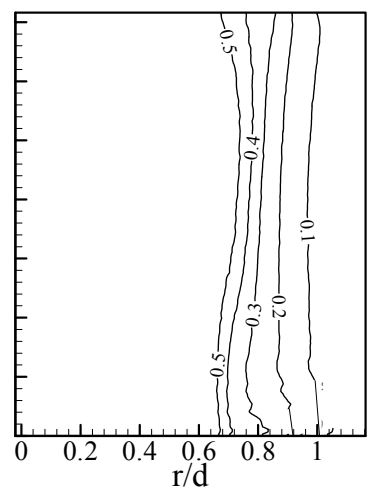

1(e)

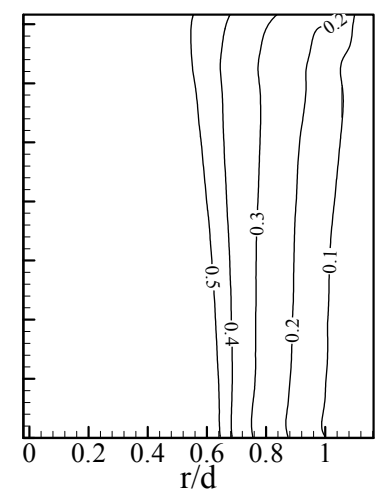

1(f)
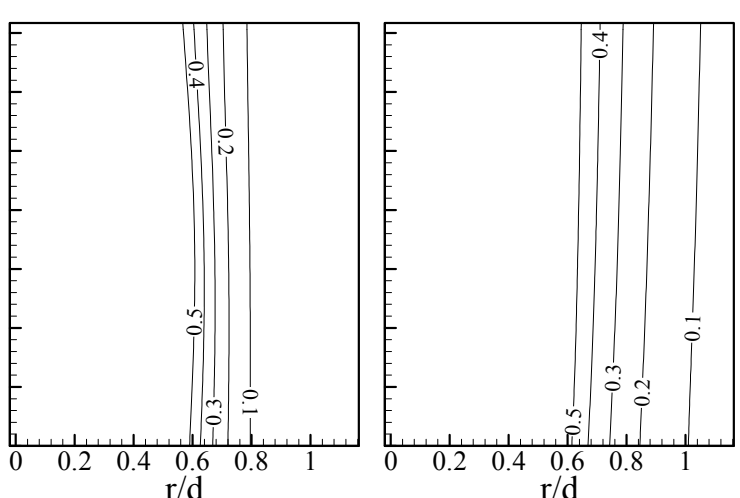

2(f)

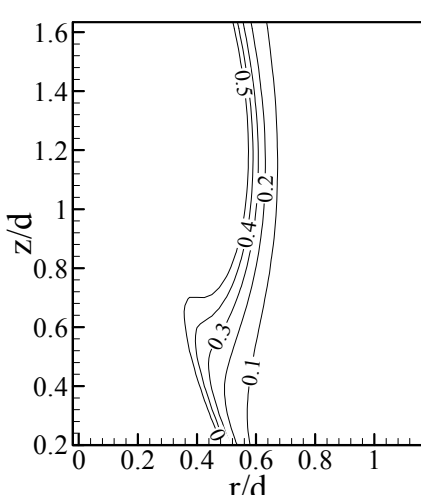

2(a)

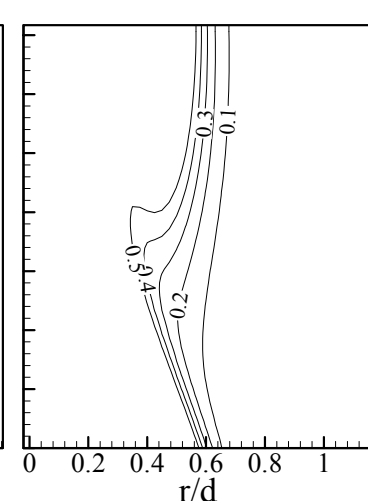

2(b)

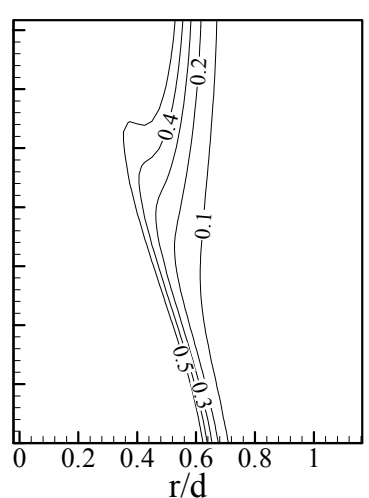

2(c)

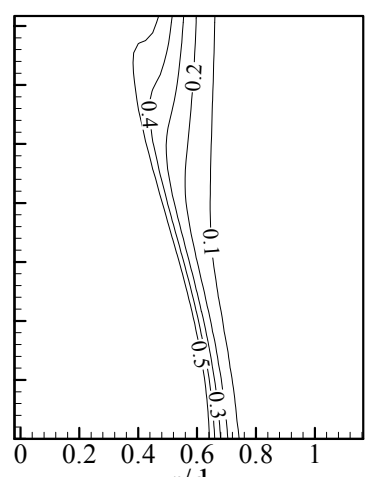

2(d)

2(e)

Figure 3. Measured (1) and Computed (2) Helium mole fraction contours during transition from Earth to microgravity at $\operatorname{Re}=300, d=31.8 \mathrm{~mm}$, and $R i=1.52$. (a) $t=0.0 \mathrm{~s} ;$ (b) $t=1 / 60 \mathrm{~s} ;$ (c) $t=2 / 60 \mathrm{~s} ;$ (d) $\mathrm{t}=3 / 60 \mathrm{~s} ;$; (e) $\mathrm{t}=13 / 60 \mathrm{~s} ;$ (f) $\mathrm{t}=2.0 \mathrm{~s}$. Measurements are taken from Yep et $\mathrm{al}^{11}$. 


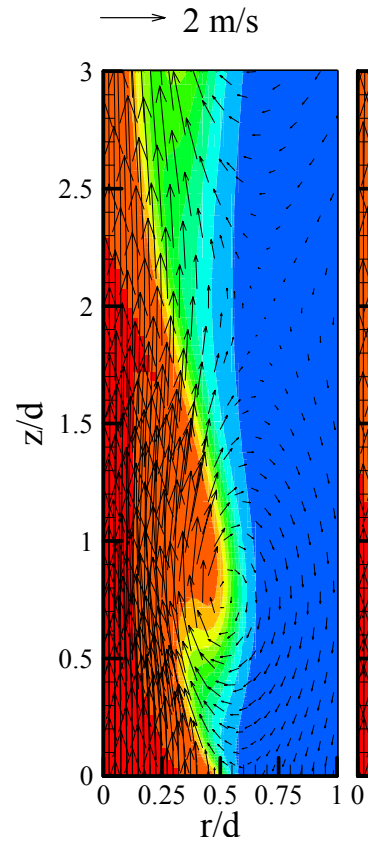

(a)

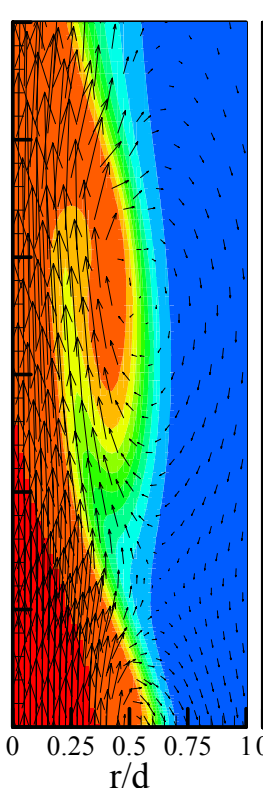

(b)

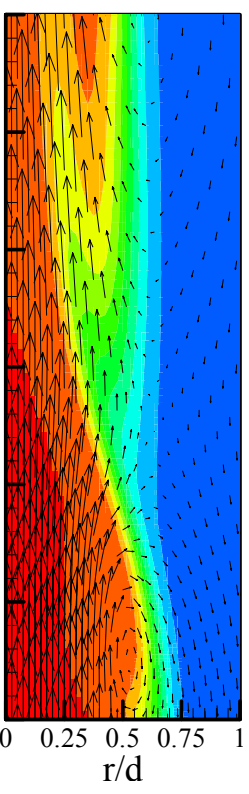

(c)

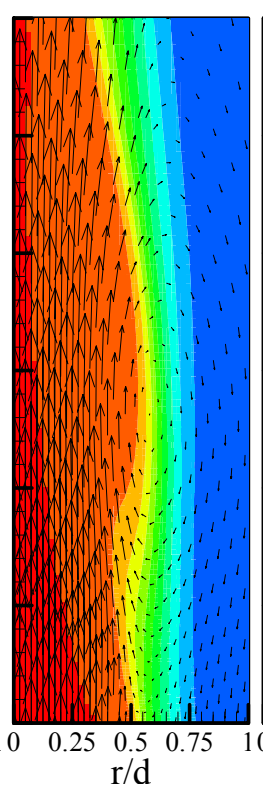

(d)

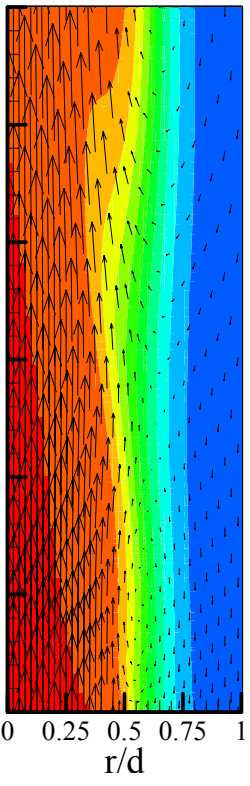

(e)

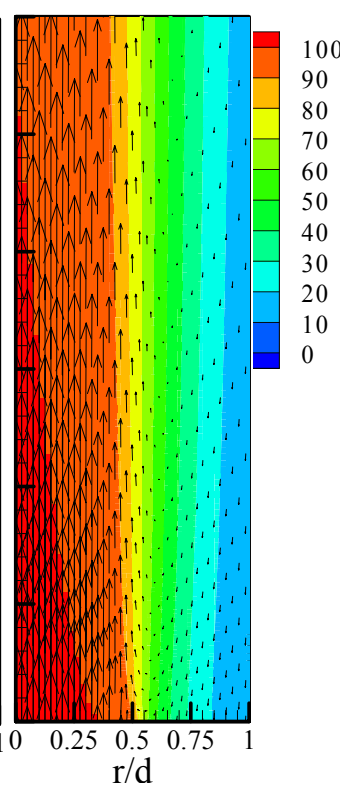

(f)

Figure 4. Contours of helium mole percentage superimposed by velocity vectors during transition from Earth gravity to microgravity (left to right) for (a) $t=0.0 \mathrm{~s} ;$ (b) $t=2 / 60 \mathrm{~s} ;$ (c) $t=4 / 60 \mathrm{~s} ;$ (d) $t=8 / 60 \mathrm{~s} ;$ (e) $t=10 / 60 \mathrm{~s}$; (f) $t=2.0 \mathrm{~s}$. Note that gravity was removed at $t=0.0$ sec.

Temporal Evolution of Axial Velocity and Concentration fields

Figure 5 shows time trace plots of helium mole percentage and axial velocity at selected axial and radial locations during the change from Earth to micro gravity conditions. Near the jet exit at $\mathrm{z} / \mathrm{d}=0.15$, the concentration at the jet center is constant at $100 \%$ throughout. In contrast, the axial velocity at the jet center fluctuates between $2.3 \mathrm{~m} / \mathrm{s}$ and $2.6 \mathrm{~m} / \mathrm{s}$ in Earth gravity. With the initiation of microgravity conditions, the axial velocity initially decreases and then reaches a steady value of $2.2 \mathrm{~m} / \mathrm{s}$ within $0.1 \mathrm{~s}$ of the change in gravity. In the entrainment region at $\mathrm{r} / \mathrm{d}=0.45$, the helium mole percentage in Earth gravity varied between $45 \%$ and $94 \%$. During transition to microgravity, the concentration peaks to $100 \%$ within $0.08 \mathrm{~s}$. Afterwards, the flow oscillates until $\mathrm{t}=0.2 \mathrm{~s}$ with gradual decrease in the amplitude. Steady state was reached at $\mathrm{t}=0.3 \mathrm{~s}$ with helium concentration reaching a value of $95 \%$. Correspondingly, the axial velocity fluctuations in Earth gravity diminish in microgravity, reaching a steady axial velocity of $0.4 \mathrm{~m} / \mathrm{s}$. Near the jet boundary at $\mathrm{r} / \mathrm{d}=0.6$, low amplitude oscillations in the concentration level were observed in Earth gravity. Helium mole percentage reached a peak value of $70 \%$ within $0.1 \mathrm{~s}$ after change of gravity. Subsequently, low Amplitude concentration oscillations were observed till $\mathrm{t}=0.4 \mathrm{~s}$ before a steady value of $52 \%$ was reached at $\mathrm{t}=$
$1.0 \mathrm{~s}$. Axial velocity fluctuations at this radial location in Earth gravity show troughs with negative velocity indicating flow recirculation. With the initiation of microgravity, the flow stabilized and reached a near zero axial velocity.

At a downstream location of $\mathrm{z} / \mathrm{d}=2.0$, the helium concentration at $\mathrm{r} / \mathrm{d}=0.0$ remained constant throughout at $100 \%$ level. However, high amplitude oscillations are evident in axial velocity ranging between $2.4 \mathrm{~m} / \mathrm{s}$ and $4.6 \mathrm{~m} / \mathrm{s}$ in Earth gravity. With the initiation of microgravity, the fluctuations diminish within $\mathrm{t}=0.5 \mathrm{~s}$ and reach steady conditions with an axial velocity of $2.1 \mathrm{~m} / \mathrm{s}$. At $\mathrm{r} / \mathrm{d}=0.45$, high amplitude concentration oscillations are observed in Earth gravity with helium concentration reaching peak levels of $95 \%$ during transition to microgravity and then reaching steady state within 0.6s. At $\mathrm{r} / \mathrm{d}=0.6$, helium reaches peak concentration level of $60 \%$ during transition to microgravity and reaches steady condition with $56 \%$ concentration after $0.6 \mathrm{~s}$. The velocity fluctuations at the above radial locations depict a similar flow behavior as already observed at the upstream location.

The above observed features are in excellent agreement with time traces of concentration fields obtained by Yep et al. ${ }^{11}$ using the $2.2 \mathrm{sec}$ drop tower. The jet expanded in microgravity and flow oscillations in concentration field existed for a brief period before attaining steady 

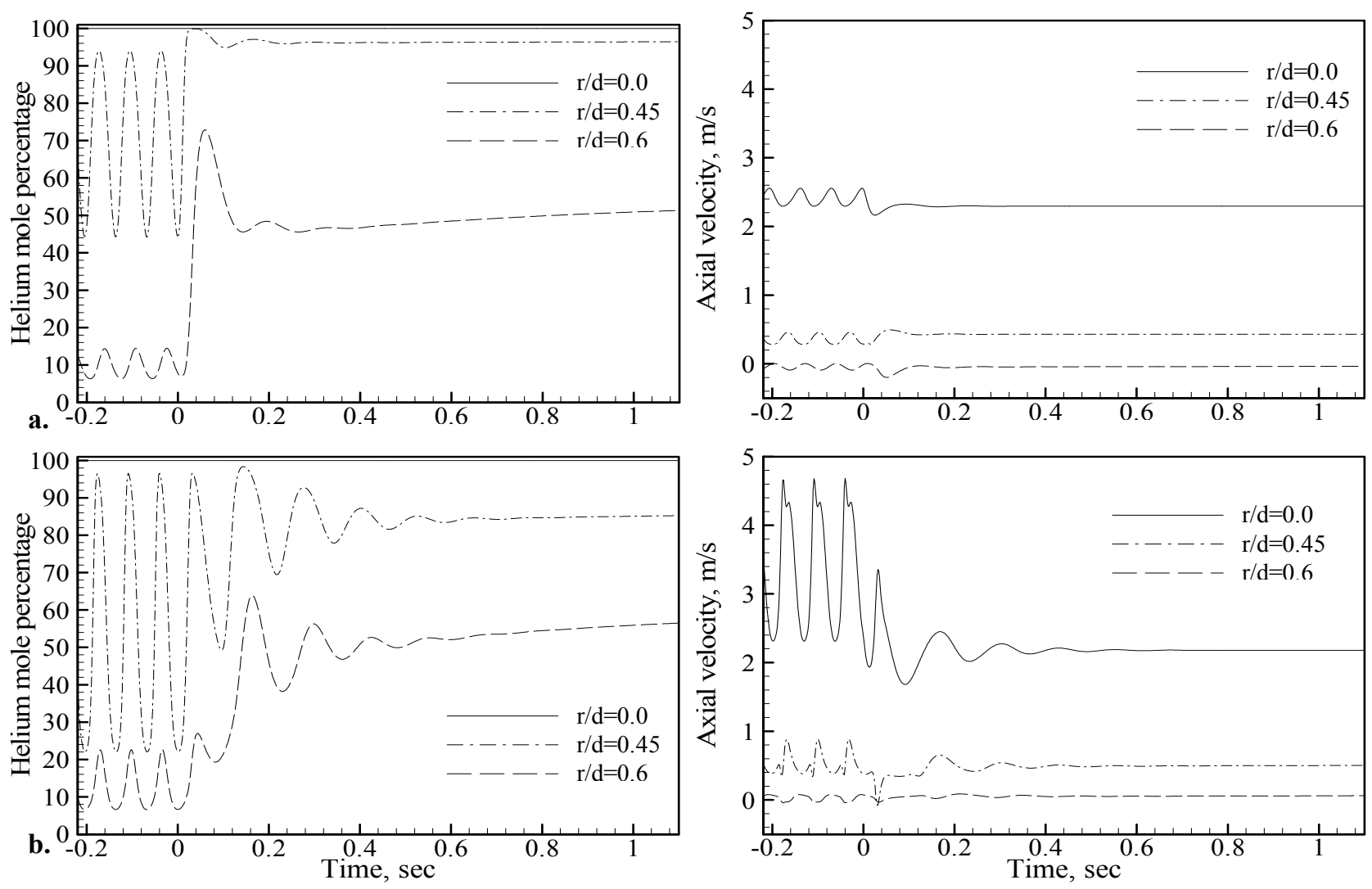

Figure 5. Time traces of helium mole percentage and axial velocity during transition from Earth gravity to microgravity at a) $\mathrm{z} / \mathrm{d}=\mathbf{0 . 1 5}$, and b) $\mathrm{z} / \mathrm{d}=\mathbf{2 . 0}$.

state conditions. Observe that an upstream location reaches steady state prior to the downstream location. Coupling between the concentration and velocity fields is explained next using radial profiles near the jet exit $(\mathrm{z} / \mathrm{d}=0.15)$ at Earth and microgravity conditions to focus on the buoyancy effects in the jet shear layer.

Figures 6 and 7 depict the radial profiles of normalized axial velocity and helium mole percentage at $\mathrm{z} / \mathrm{d}=0.15$ for Earth and microgravity conditions. Note that the axial velocity has been normalized with the jet exit velocity at respective radial locations. In Earth gravity, the normalized axial velocity profile shows a gradual increase from the jet core towards the tube wall indicating greater acceleration in the outer region of the jet. The peak normalized axial velocity corresponds to a value of 1.2 at $\mathrm{r} / \mathrm{d}=0.4$, a location coinciding with the sudden drop in concentration. Thus, we observe that buoyancy causes large axial velocity gradients and unstable density stratification near the jet shear layer, leading to the entrainment of ambient fluid into the primary jet to produce the oscillatory flow.
In microgravity, a flat normalized axial velocity profile with a minor dip near the tube wall is noticed (Ref. figure 7). The concentration changes gradually as helium diffuses into the ambient environment. Results show that the lack of buoyant acceleration in the jet shear produces a non-oscillatory flow. The next section provides quantitative details relating to the vortex movement and frequency and amplitude of flow oscillations with changes in the gravitational forcing.

\section{Flow Structure: Dependence on Gravitational Forcing}

In this section, the analysis has been limited only to the vortex movement and the frequency and amplitude of flow oscillations as functions of the gravitational forcing. Figure 8 illustrates the convection velocity of the vortex core at different gravitational levels. For Martian gravity $(\mathrm{g} / \mathrm{go}=0.37)$, we observe that the vortex convected at a uniform acceleration with peak velocity of $0.9 \mathrm{~m} / \mathrm{s}$ at $\mathrm{z} / \mathrm{d}=3.0$. Further, in Earth gravity $(\mathrm{g} / \mathrm{go}=1.0)$, the vortex convection velocity increased at all the axial stations with a peak velocity of $1.0 \mathrm{~m} / \mathrm{s}$. 


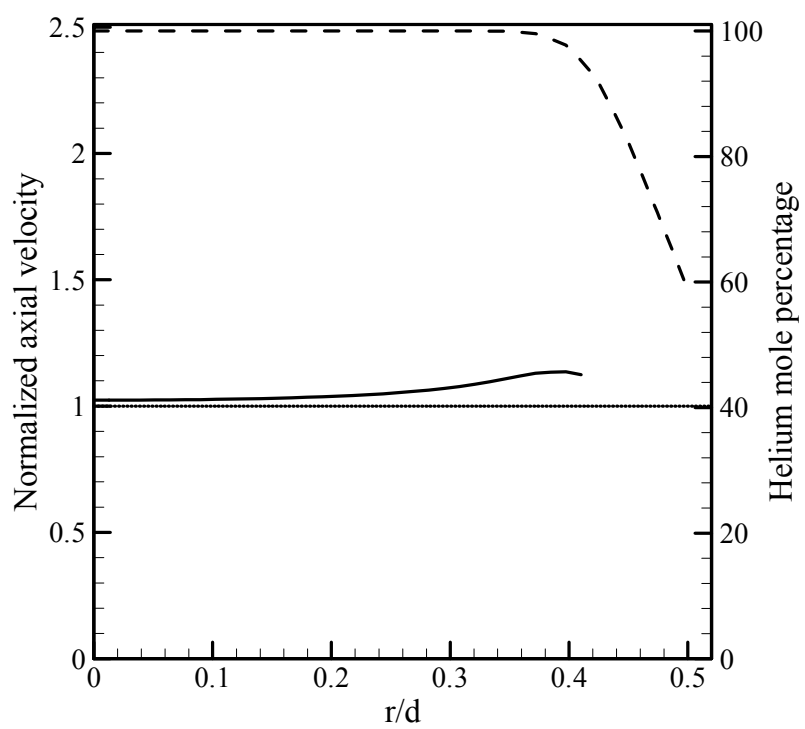

Figure 6. Helium mole percentage and normalized axial velocity profiles in Earth gravity at $\mathrm{z} / \mathbf{d}=\mathbf{0 . 1 5}$.

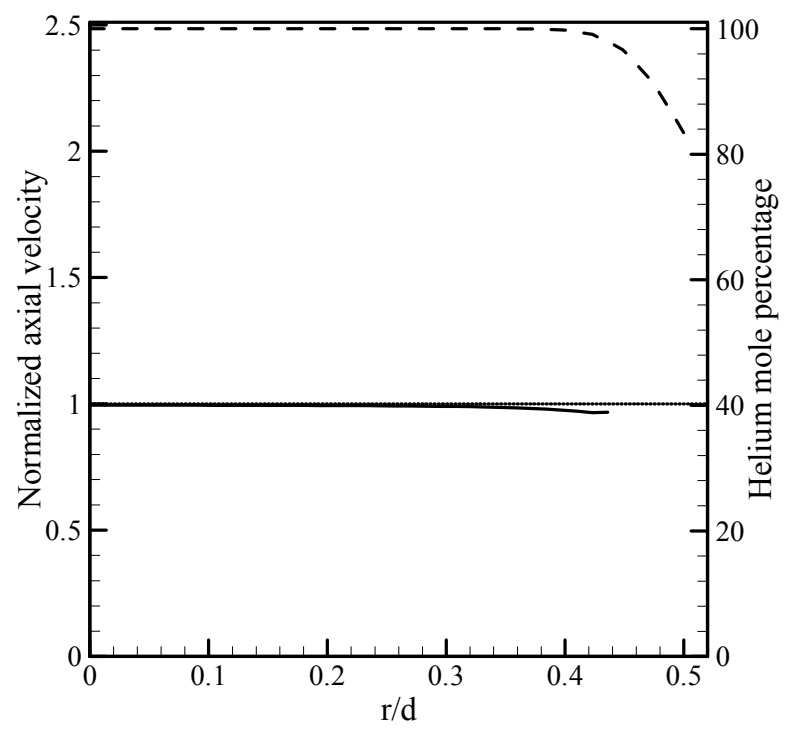

Figure 7. Helium mole percentage and normalized axial velocity profiles in microgravity at $\mathrm{z} / \mathbf{d}=\mathbf{0 . 1 5}$.

In Jupiter gravity $\left(\mathrm{g} / \mathrm{g}_{0}=2.33\right)$, the vortex convects with higher acceleration throughout the near field as shown by the steep rise of the curve, with peak velocity reaching about $2.0 \mathrm{~m} / \mathrm{s}$. Notice that with increase in buoyancy, the vortex convection velocity increases throughout the near field. Accordingly, the entrainment rate, which is a function of the vortex convection velocity, would also increase at higher gravitational levels. Higher entrainment rate leads to enhanced mixing and contamination of the jet core in the downstream region.

Figures 9 and 10 show plots explaining the variation

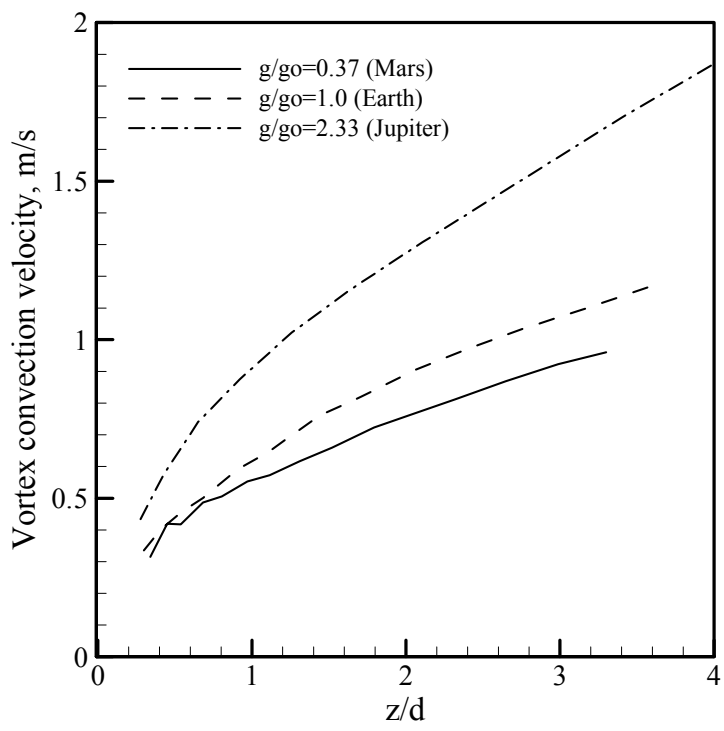

Figure 8. Vortex convection velocity at various gravitational levels.

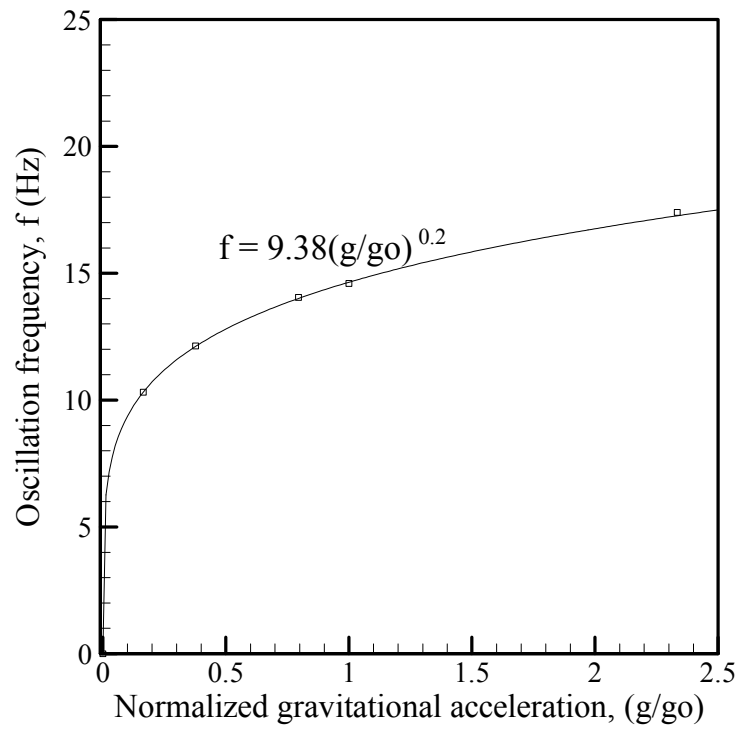

Figure 9. Oscillation frequency Vs normalized gravitational acceleration.

of frequency and amplitude of flow oscillation with the gravitational level. The best fit resulted in the relationship between oscillation frequency and gravitational acceleration as $\mathrm{f}=9.38(\mathrm{~g} / \mathrm{go})^{0.2}$. The normalized amplitude of the jet oscillatory structure in Figure 10 shows dependence on the gravitational level as well. The amplitude refers to that of the concentration wave and normalization is done using amplitude at Earth gravity. An increase in amplitude with the rise in gravitational level suggests that buoyancy enhances wrapping of the ambient fluid into the jet flow field to affect the entrainment and mixing characteristics in the oscillating shear layer. 


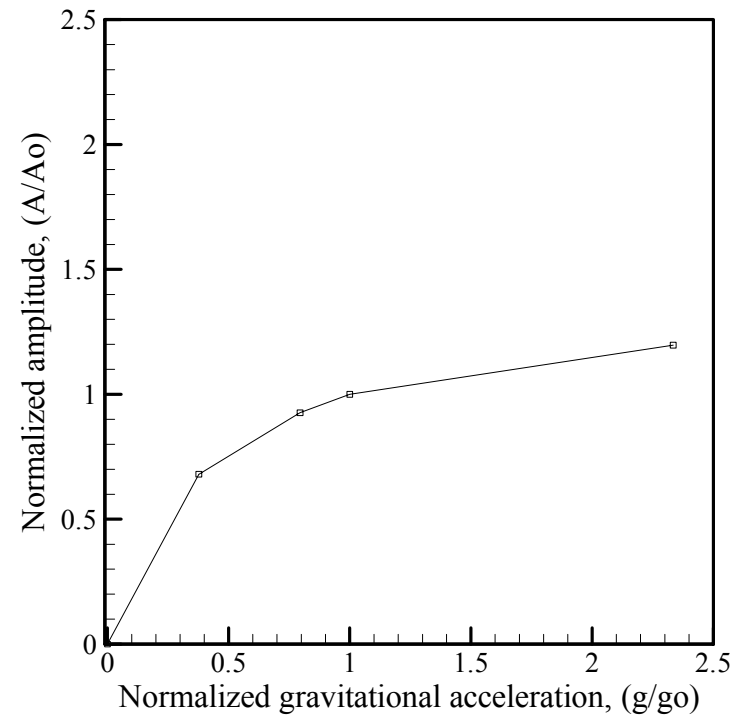

Figure 10. Normalized concentration amplitude Vs normalized gravitational acceleration at $\mathrm{z} / \mathrm{d}=\mathbf{1 . 0}$ and $\mathrm{r} / \mathrm{d}=\mathbf{0 . 4 5}$.

\section{CONCLUSIONS}

Numerical simulations of a helium jet injected into quiescent air environment were performed to describe the effects of buoyancy in the jet flow field. The interaction of the velocity and the concentration fields was presented by simultaneous visualizations at Earth and microgravity conditions. Results indicated gravity induced global oscillations occurring in the near field of the jet at Earth gravity. Further, the gradual weakening and diminishing of oscillations during transition from Earth to microgravity confirmed that flow oscillations are buoyancy induced. Temporal plots of velocity and concentration fields depict that oscillations existed for a brief period during transition from Earth to microgravity. The jet width increased in microgravity and the upstream locations of the jet reached steady state prior to the downstream locations. Radial profiles of normalized axial velocity and concentration fields near the jet exit illustrated the jet diffusion and entrainment processes characterizing the instability. It was found that the flow oscillation frequency and amplitude increase with the rise in the gravitational level.

\section{ACKNOWLEDGEMENTS}

This work was supported by Physical Sciences Division of NASA's office of Biological and Physical research under grant NAG 3-2388.

\section{NOMENCLATURE}

Tube Inside Diameter ( $\mathrm{mm})$

frequency $(\mathrm{Hz})$

g Accelaration due to gravity $\left(\mathrm{m} / \mathrm{s}^{2}\right)$

$\mathrm{M}_{\mathrm{w}} \quad$ Molecular Weight $(\mathrm{Kg} / \mathrm{Kmole})$

$\mathrm{P} \quad$ Pressure $(\mathrm{Pa})$

R Universal Gas Constant $(\mathrm{J} / \mathrm{KgK})$

Re Jet Reynolds Number

Ri Jet Richardson Number

$\mathrm{T} \quad$ Absolute Temperature(K)

$\mathrm{V} \quad$ Average jet exit velocity $(\mathrm{m} / \mathrm{s})$

Y Mole Fraction

Greek Symbols

$\mu \quad$ Viscosity $(\mathrm{Kg} / \mathrm{m}-\mathrm{s})$

$\rho \quad \operatorname{Density}\left(\mathrm{Kg} / \mathrm{m}^{3}\right)$

Subscripts

$\begin{array}{ll}\text { air } & \text { Air } \\ \text { he } & \text { Helium } \\ \text { i } & \text { Species i } \\ \text { j } & \text { Species j } \\ \infty & \text { Ambient fluid }\end{array}$

\section{REFERENCES}

${ }^{1}$ Fay, J.A., (1973), "Buoyant plumes and wakes," Annual Review of Fluid Mechanics, Vol. 5, pp. 151160.

${ }^{2}$ Morton, B.A., (1959), "Forced plumes," Journal of Fluid Mechanics, Vol. 5, pp. 151-159.

${ }^{3}$ List, E.L., (1982), "Turbulent Jets and plumes," Annual Review of Fluid Mechanics, Vol. 14, pp. 189.

${ }^{4}$ Papanicolaou, P.A., and List, E.L., (1988), "Investigations of Round Vertical Turbulent Buoyant Jets," Journal of Fluid Mechanics, Vol. 195, pp. 342391.

${ }^{5}$ Dai, Z., Tseng, L.K., and Faeth G.M., (1994), "Structure of round, fully developed, buoyant turbulent plumes," Journal of Heat Transfer, Vol. 116 , pp. 409-417.

${ }^{6}$ So, R.M.C., Zhu, J.Y., Otugen, M.V., and Hwang, B.C., (1990), "Some Measurements in a Binary Gas Jet," Experiments in Fluids, Vol. 9, pp. 273-284. 
${ }^{7}$ Subbarao, E.R., and Cantwell B.J., (1992), "Investigation of a co-flowing buoyant jet: experiments on the effects of Reynolds number and Richardson number," Journal of Fluid Mechanics, Vol.245, pp. 69-90.

${ }^{8}$ Cetegen, B.M., and Ahmed, T.A., (1993), "Experiments on the periodic instability of buoyant plumes and pool fires," Combustion and Flame, Vol. 93, pp. 157-184.

${ }^{9}$ Cetegen, B.M., and Kasper, K.D., (1996), "Experiments on the oscillatory behavior of buoyant plumes of helium and helium-air mixtures," Physics of Fluids, Vol. 8, pp. 2974-2984.

${ }^{10}$ Cetegen, B.M., (1997), "Measurements of Instantaneous Velocity field of a Non-Reacting Pulsating Buoyant Plume by Particle Image Velocimetry," Combustion Science and Technology, Vol. 123, pp. 377-387.

${ }^{11}$ Yep, T.W., and Agrawal, A.K., (2003), "Gravitational Effects on near field flow structure of Low density gas jets," AIAA Journal, Vol. 41, pp. 1973-1979.

${ }^{12}$ Pasumarthi, K.S., and Agrawal, A.K., (2003), "Schlieren Measurements and Analysis of Concentration Field in Self-Excited Helium Jets," Physics of Fluids, Vol. 15 (In Press).

${ }^{13}$ Mell, W.E., McGrattan, W.B., Baum, H.R., (1996), "Numerical Simulation of Combustion in Fire Plumes," Proceedings of the Combustion Institute, Vol. 26, pp. 1523-1530.

${ }^{14}$ Hamins, A., Yang, J.C., Kashiwagi, T., (1992), “An Experimental Investigation of the Pulsating Frequency of Flames," Proceedings of the Combustion Institute Vol. 24, pp. 1695-1705.

${ }^{15}$ Soteriou, M.C., Dong, Y., Cetegen, B.M., (2002), "Lagrangian simulation of the unsteady near field dynamics of planar buoyant plumes," Physics of Fluids Vol. 14, pp. 3118-3140.

${ }^{16}$ Bird, R.B., Stewart, W.E., Lightfoot, E.N., (1960), "Transport Phenomena, $1^{\text {st }}$ edition. Wiley, New York.

${ }^{17}$ Fluent 6.0, User's Guide, Fluent Inc. Lebanon, NH 1-4 (2003).
${ }^{18}$ Gambit 2.1, User's Guide, Fluent Inc. Lebanon, NH 1-4 (2003). 